\title{
MITTEILUNGEN DER GESELLSCHAFT FÜR INFORMATIK / 221. FOLGE
}

\author{
Gesellschaft für Informatik e.V. (GI), Wissenschaftszentrum, Ahrstraße 45, 53175 Bonn, Tel.: 0228/302-145, Fax 0228/302-167, \\ e-mail: gs@gi.de, Server: http://www.gi.de \\ Geschäftsführung: Dr. Peter Federer, e-mail: peter.federer@gi.de, Tel.: -145 \\ Stellvertreterin der Geschäftsführung: Cornelia Winter, e-mail: cornelia.winter@gi.de, Tel.: -147 \\ Stellvertreter des Geschäftsführers: Alexander Rabe, e-mail: alexander.rabe@gi.de \\ Sekretariat: Monika Schulte, e-mail: monika.schulte@gi.de, Tel.: -145 \\ Mitgliederwerbung: Ludger Porada, e-mail: ludger.porada@gi.de, Tel.. -146 153 S \\ Mitgliederverwaltung: Tanja Diegeler, e-mail: tanja.diegeler@gi.de, Tel.. - -149, Karola Schmitt, e-mail: karola.schmitt@gi.de, Tel.. -151 \\ ITK: Christopher Schiller, e-mail: christopher.schiller@gi.de, Tel. - -156
}

\section{i \\ Kompetenzzentrum Deutschland}

Organisiert vom Wirtschaftsrat Deutschland fand im März im BCC in Berlin das sogenannte „Kompetenzzentrum Deutschland“ unter dem Motto „Wachstums- und Beschäftigungstreiber Internet" unter Beteiligung der GI mit über 1000 Teilnehmern statt. Netzpolitik ist Wirtschaftspolitik - in diesem Sinne wurden gemeinsam mit Vizekanzler und Bundeswirtschaftsminister Dr. Philipp Rösler und Bundesinnenminister Dr. Hans-Peter Friedrich MdB sowie weiteren Persönlichkeiten aus Politik und Wirtschaft die Handlungsnotwendigkeiten aufgezeigt und Lösungsansätze identifiziert. GI-Vizepräsident Peter Liggesmeyer vertrat die GI auf einer Podiumsdiskussion zum Thema Big Data - Big Chances, u. a. mit Staatssekretärin Rogall-Grothe (BMI) und Peter Tauber (MdB). Die GI ist seit Sommer 2012 in einigen Bundesfachkommissionen des Wirtschaftsrates zu verschiedenen Fachthemen als Gast aktiv.

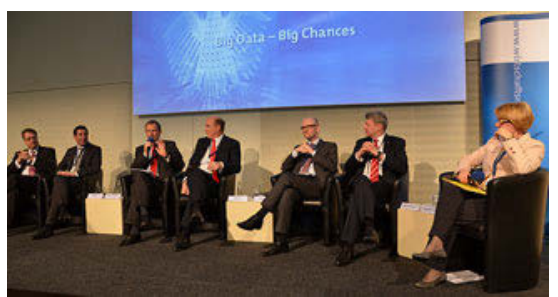

GI-Vizepräsident Liggesmeyer (2. v. rechts) bei der Podiumsdiskussion zu "Big Data"
Strategische Kooperation zwischen Gl und eco besiegelt

Die Gesellschaft für Informatik e.V. (GI) und der Verband der Internetwirtschaft e.V. eco haben im März 2013 eine strategische Partnerschaft vereinbart. Gemeinsam verfolgen GI und eco folgende Ziele:

- Entwickeln von Maßnahmen gegen den Fachkräftemangel

- Förderung der Sichtbarkeit von IT/Informatik in der politischen Willensbildung und im gesellschaftlichen Kontext

- Unterstützung der gemeinsamen Aus- und Weiterbildung von IT-Nachwuchs und Fach- und Führungskräften

- Förderung der Bildung von Forschungskonsortien zwischen Hochschulen und Internetwirtschaft

„Der Fachkräftemangel in der Informatik wird immer mehr $\mathrm{zu}$ einem ernstzunehmenden Problem der deutschen Wirtschaft", sagte GI-Präsident Oliver Günther anlässlich der Vertragsunterzeichnung in Berlin. „Gemeinsam mit der Internetwirtschaft müssen wir dem entgegenwirken und darüber hinaus auf eine kontinuierliche Weiterbildung unserer Fachleute achten“, so Günther. Als eine Maßnahme könnte die engere Kooperation zwischen Hochschulen und der Internetwirtschaft dienen.
Darüber hinaus wollen GI und eco ein nachhaltig positives Bild der Informatik und der Informatik-Fachleute in der Öffentlichkeit etablieren und Verständnis und Interesse für eine Beschäftigung in der Informatik und der Internetwirtschaft wecken. „IT und Internetwirtschaft in Deutschland brauchen eine Sichtbarkeit auf politischer und gesellschaftlicher Ebene, die ihrer Bedeutung für die deutsche Wirtschaft gerecht wird. Dafür wollen wir uns gemeinsam einsetzen", sagt eco Vorstandsvorsitzender Prof. Michael Rotert.

„Außerdem eröffnet sich durch diese Kooperation unserem ITNachwuchs die Chance, bereits früh im Studium in den Unternehmen des eco-Verbandes praktische Erfahrungen zu sammeln“, sagte Günther. Im Gegenzug habe eco die Möglichkeit, mit Informatikstudierenden in Kontakt zu kommen und sie als Mitarbeiter zu gewinnen.

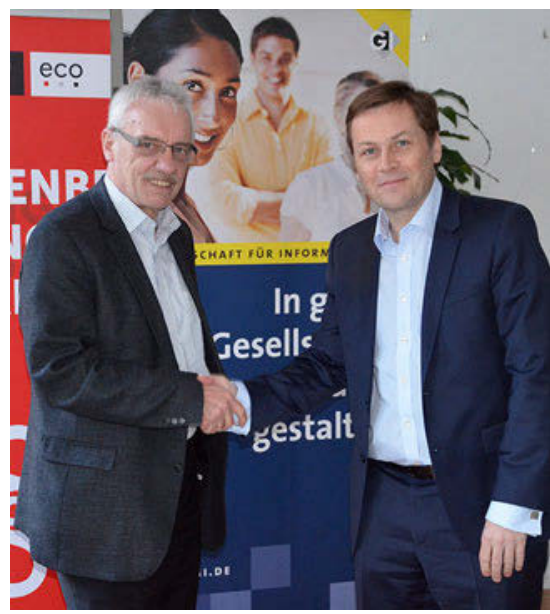

eco-Vorsitzender Rotert (links) und GIPräsident Günther nach der Vertragsunterzeichnung

DOI $10.1007 / 500287-013-0708-x$

Die Fortsetzung der Mitteilungen folgt auf Seite 343. 